\title{
Integración de las nuevas tecnologías en el manejo de las aguas subterráneas
}

\author{
Integration of new technologies to groundwater management
}

Fecha de entrega: 13 de enero 2012

Fecha de aceptación: 10 de octubre 2012

\section{Armando Hernández, Eric Cabrera y Modesto Gómez}

Centro de Investigaciones Hidráulicas, Facultad de Ingeniería Civil, Instituto Superior Politécnico José Antonio Echeverría, Calle 114, núm. 11901 entre 119 y 127, Marianao, 19390, La Habana, Cuba, ahernandez@cih.cujae.edu.cu, ecabrerae@cipp.minbas.cu, modesto@cih.cujae.edu.cu

Se muestran las características de la Plataforma Modular Integrada PMI, elaborada por uno de los autores para el manejo de los recursos hidráulicos subterráneos. Se vincula la adquisición automatizada de datos en tiempo real, el procesamiento sobre una plataforma SIG, el uso de modelos de simulación y las técnicas de optimización para la gestión óptima de acuíferos y operación automatizada de las obras de captación. Se presentan las características y experiencias de aplicación en la modelación de acuiferos cubanos con el software AQÜIMPE, basado en el método de elementos finitos y que utiliza el triángulo cuadrático como elemento de discretización, lo cual permite utilizar elementos volumétricos representativos en acuiferos cársticos, como medios porosos equivalentes. Se muestra el procedimiento utilizado en Cuba encaminado a evaluar los recursos hídricos subterráneos utilizando el modelo de simulación del acuifero. Del trabajo se concluye que la utilización de modelos de administración para definir políticas óptimas de explotación, una vez definidos los recursos de explotación con un modelo regional de flujo según horizontes de planeamiento, es necesario vincularlo a las obras de captación mediante refinamiento de mallas y factores que consideren los efectos locales dependientes del gasto especifico. Como caso de estudio de implementación de la PMI y de las nuevas tecnologías que en ella se contemplan, se presentan los resultados de la aplicación en un acuifero costero que abastece a la ciudad de La Habana.

Palabras clave: acuiferos, modelación matemática, modelos de administración del agua subterránea, sistemas integrados para la gestión de acuiferos
Characteristics of the Modular Integrated Platform PMI, compiled by one of the authors, are shown for the management of groundwater resources. The automated data acquisition in real-time processing on a GIS platform, the use of simulation models and optimization techniques for optimal management of aquifers and automated operation of the headworks are linked. The characteristics and experiences of applications in modeling with the software AQÜIMPE in Cuban aquifers, are presented. AQÜIMPE is based on the finite element method using quadratic triangle as discretization element, allowing representative volumetric elements used in karstic aquifers, such as porous equivalents media. The procedure used in Cuba aimed at evaluating groundwater resources using the simulation model of the aquifer, is described. From this study, it is concluded that the use of management models to define optimal operating policies, once operating resources are defined with a regional model of flow as planning horizons, must link it to the headworks through mesh refinements and factors considering local effects dependent on specific flow use. As a case study, the implementation of the PMI and new technologies contemplated therein, are the results of the application in a coastal aquifer which supplies the city of La Havana.

Keywords: aquifers, mathematical modelling, groundwater administration model, integrated systems for managing aquifers 


\section{Introducción}

En muchas regiones del mundo la principal fuente de abasto de agua la constituyen los sistemas acuíferos. En el caso particular de Cuba, la capital La Habana con una población alrededor de los 3 millones de habitantes, se abastece en un $99 \%$ de las aguas subterráneas.

Es usual en nuestro país que las empresas que se dedican al diseño, construcción, rehabilitación y operación de las redes de acueducto, utilicen los datos en cuanto a disponibilidad del recurso hídrico que otras empresas le suministran y en ocasiones prioricen garantizar la demanda actual sin preocuparse de lo que puede suceder con la fuente de abasto en un horizonte de planeamiento a largo plazo. Lo anterior puede conllevar a afectaciones graves en el suministro y a costos elevados al tener que utilizar fuentes y métodos de distribución alternativos.

En adelante se exponen algunas tecnologías de avanzada que permiten captar las bases de datos aportadas por diferentes métodos de estudio de las aguas subterráneas, procesarlas y suministrarlas a los sistemas informáticos para mediante la modelación matemática, integrarlas y obtener respuestas que permitan a los especialistas proponer soluciones a los tomadores de decisión para la definición de políticas de operación.

\section{Plataforma modular integrada PMI para la gestión del agua subterránea como recurso}

Hasta el presente, la aplicación conjunta de la modelación matemática, los sistemas de información geográfica y las tecnologías de control y supervisión de procesos en tiempo real, para el análisis y gestión de los sistemas de recursos hidráulicos, ha sido abordada de manera parcial. En este trabajo, se muestra la necesidad de emplear una Plataforma Modular Integrada PMI para la gestión del agua subterránea como recurso, basada en el empleo interrelacionado de estas herramientas. Dentro de los factores que favorecen la implementación de la plataforma propuesta y realizan una contribución a la gestión del agua subterránea se encuentran: la posibilidad de su instalación por etapas, su enfoque modular, la concepción de una base de datos única que posibilita el uso compartido de la información, y la utilización de diferentes esquemas de captación de las variables, todo ello articulado desde una supervisión centralizada del proceso, en tiempo real.
En Gómez (2009), se ejemplifica en dos casos de estudio la aplicación de la plataforma propuesta. Estos demuestran la validez del método de integración planteado y del empleo de la nueva versión del modelo matemático de simulación AQÜIMPE para el pronóstico de la política de explotación del recurso.

El diseño modular de la PMI permite establecer opciones para su implantación gradual que constituyen una vía factible de completamiento de las tareas de gestión de los recursos hidráulicos para las entidades encargadas de administrarlos. Esta concepción de implantación, no exige la aplicación total de todos los módulos de la PMI, desde el propio comienzo, sino que permite incorporar en cada etapa, nuevos elementos para la gestión, que complementan los precedentes, en función de los recursos disponibles para inversión.

La experiencia acumulada en la operación de sistemas hidráulicos y la premisa de realizar bajas inversiones que sean factibles de acometer por las entidades encargadas de su administración y representen una vía efectiva para obtener resultados progresivos en el control de los recursos hidráulicos, orientan los escalones a establecer en una secuencia como la que a continuación se detalla:

1- Calibración de los modelos matemáticos necesarios para la gestión de la operación, a partir de las bases de datos disponibles. Comienzo de la operación, basado en pronósticos realizados a través de los modelos de simulación del sistema $(\mathrm{SIG}+\mathrm{MM})$ con la toma de decisiones en tiempo diferido y entrada de datos no automatizada. Representa la fase que requiere la menor inversión. El procedimiento lógico de trabajo, esquematizado en la Figura 1, consiste esencialmente en la conformación del marco inicial de análisis para el establecimiento de las primeras Reglas de Operación RDO, que permanecen sin variación hasta la llegada de la nueva información de campo, momento en el que se actualiza el SIG y se ejecutan los modelos matemáticos para decidir las próximas RDO. La vía de captación de datos es la tradicional, por teclado.

2- La aplicación parcial de la PMI (SIG + MM + Sensores con Registradores de Variables) para operación y toma de decisiones en tiempo diferido con entrada de datos parcialmente automatizada se ilustra en la Figura 2. Representa el escalón intermedio en cuanto a alcance de la gestión y requerimientos de inversión. Se introduce, 
adicionalmente, el modo de captación de datos de tiempo diferido, lo que permite actualizar de manera periódica la base de datos de la PMI a través de la ejecución del módulo WinRM y la descarga de las memorias de registro.

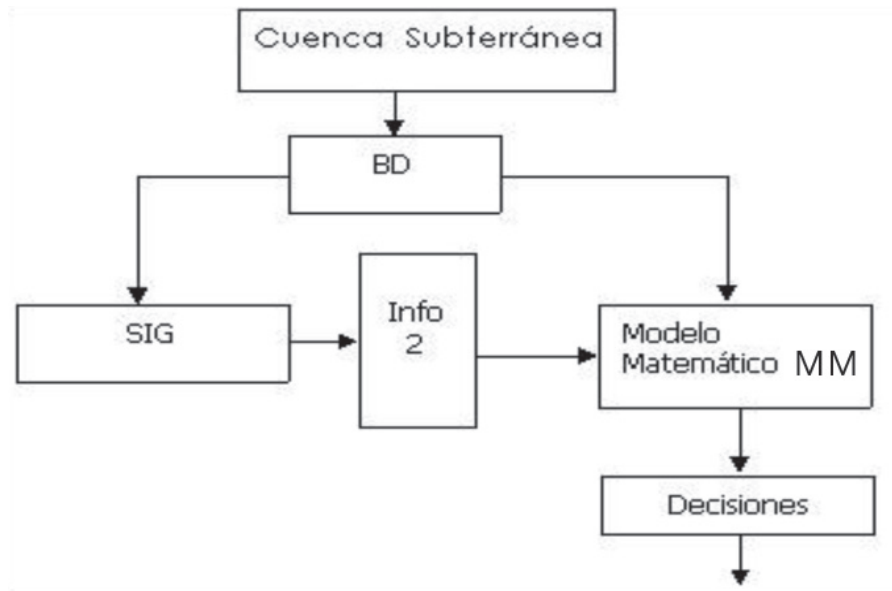

Figura 1: Procedimiento lógico de trabajo asociado con el Escalón No. 1

Mejora la base informativa y permite detectar a posteriori, irregularidades ocurridas en el cumplimiento de las RDO. Tanto en este escalón, como en el tercero, el procedimiento general de trabajo para la gestión de la cuenca, es controlado por el SGO (Supervisor General de la Operación).

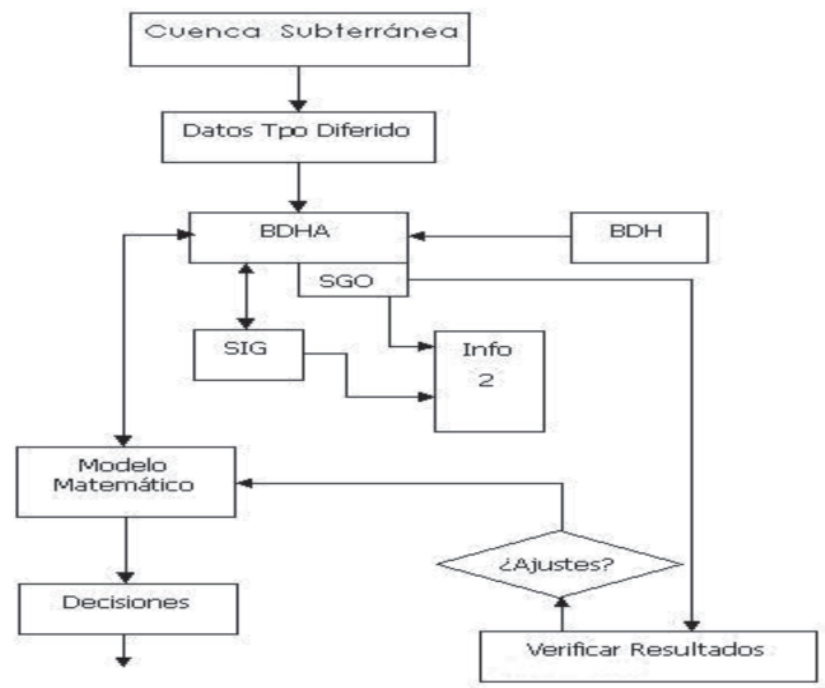

Figura 2: Procedimiento lógico de trabajo asociado con el Escalón No. 2

3- La aplicación de la PMI con todos sus componentes ( $\mathrm{SIG}+\mathrm{MM}+$ Sensores y actuadores con Telegestión y Sensores con Registradores de Variables + SCADA) con toma de información en tiempo real y diferido y operación en tiempo real, empleando la mejor estrategia para cada aplicación. Constituye el último escalón en la implantación de la PMI. Se introduce el modo de captación de datos en tiempo real, a partir de la incorporación a la PMI del sistema SCADA, con lo cual se completa la integración total de las herramientas de gestión.

El procedimiento de trabajo mostrado en la Figura 3, se enuncia a continuación:

a) Conformación del marco inicial de análisis para el establecimiento de las primeras $\mathrm{RDO}$, con arreglo a la información que se dispone al momento de la puesta en marcha de la PMI, en forma de base de datos histórica y los resultados de las corridas de los modelos de simulación y optimización para el siguiente periodo de operación;

b) Puesta en marcha y operación ininterrumpida de la automatización que permite conformar la base de datos histórica actualizada, a partir de la información de campo suministrada por el equipamiento de medición, tanto en tiempo real, como diferido. El tratamiento de esta información, así como los efectos de las acciones de control es realizado por el SCADA y el SIG, personalizado para cada aplicación;

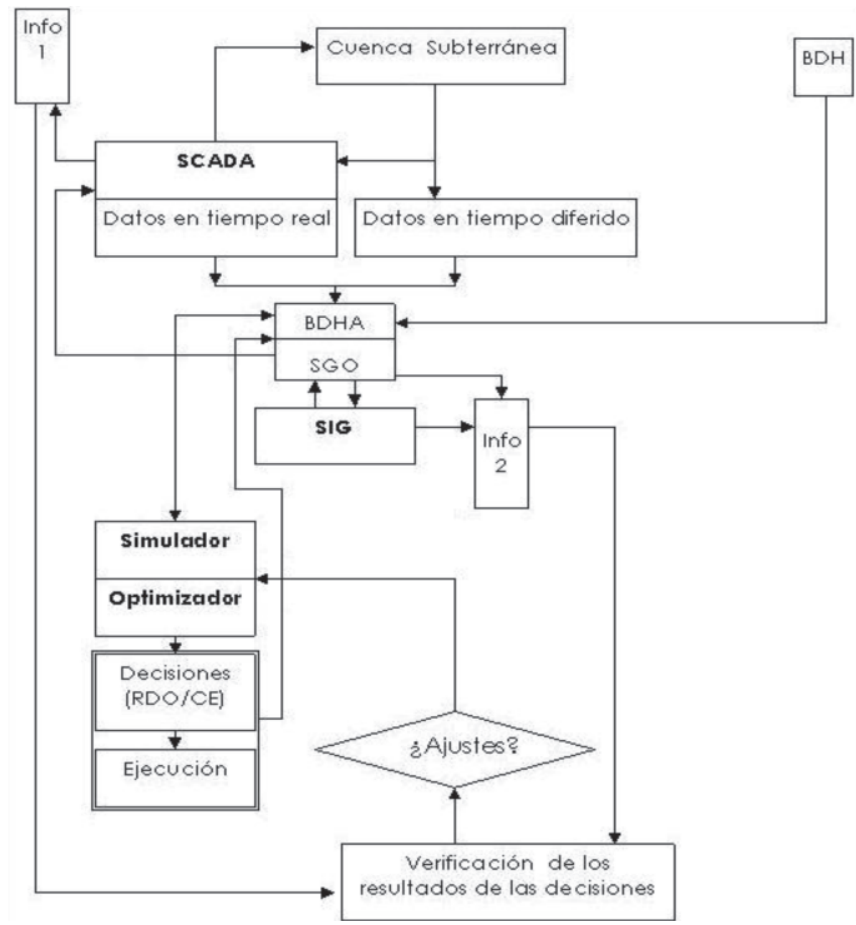

Figura 3: Procedimiento lógico de trabajo asociado con el Escalón No. 3 
c) Retroalimentación y actualización de las RDO a partir de la información en línea que se genera durante todo el periodo de operación y los resultados del contraste del análisis de la información real obtenida, con los objetivos inicialmente planteados a partir de las simulaciones realizadas. Si el análisis de la información de campo recolectada arroja resultados que se alejan de los requerimientos planteados o cuestionan el comportamiento del sistema para el siguiente periodo de operación, se ejecutan los modelos de simulación y optimización con vistas a reacomodar las RDO y ajustarlas a las nuevas condiciones.

La existencia de la PMI definida en Figura 4, permite desarrollar una gestión multilateral de la operación de los recursos hidráulicos de una región. Aun cuando se acometa la implantación de los primeros escalones, es posible garantizar los objetivos esenciales de satisfacción del usuario, economía y sustentabilidad.

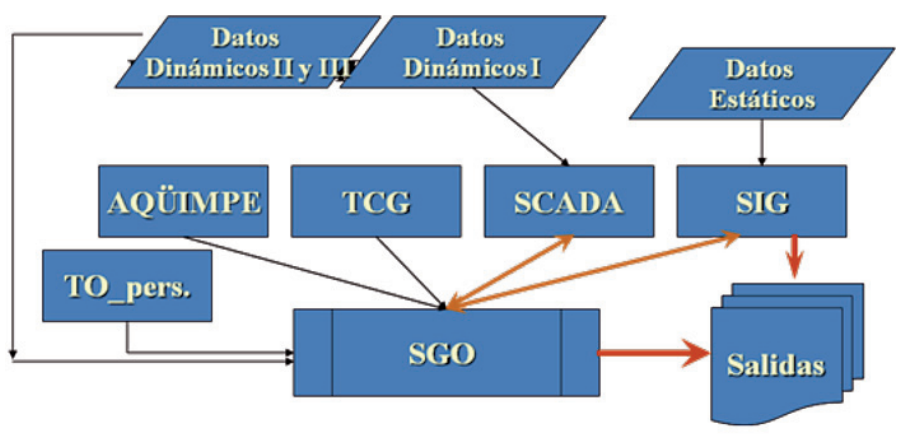

SIG: Sistema de Información Geográfica; SCADA: Sistema de Adquisición de Datos, Supervisión y Control; RDO/CE: Reglas de Operación del Sistema basado en Criterio de Especialistas, específico para la aplicación; SGO: Supervisor General de la Operación: Planifica el trabajo de los módulos; Info. 1: Datos puntuales, tablas y gráficos de: niveles dinámicos, calidad del agua de cada pozo seleccionado, gasto, presión en la descarga, horas de trabajo y variables eléctricas de cada bomba, gasto y presión en puntos de la red, volúmenes de tanques de almacenamiento, alarmas del sistema; Info. 2: Mapas, tablas y gráficos de: niveles del acuífero actuales y pronosticados, niveles de intrusión salina actuales y pronosticados, información detallada de las variables que se midan en cada pozo seleccionado, información en la red de conducción $(\mathrm{Q}, \mathrm{H})$, datos geográficos, datos de lluvia, datos de calidad del agua.

\section{Figura 4: Esquema de trabajo de la PMI}

La satisfacción del usuario en cuanto al suministro en cantidad y calidad se garantiza mediante la generación de las RDO, de tal manera que se tengan en cuenta los aspectos que más peso relativo tienen en las decisiones. Las RDO establecen los intervalos de tiempo en los que se activan las fuentes y el caudal de cada una de ellas. A partir del conocimiento anticipado del comportamiento del sistema que permiten los modelos, la decisión va cambiando según convenga para el mejor cumplimiento de los intereses de usuario. Los aspectos de la economía quedan también considerados en los párrafos anteriores en una forma directa, por cuanto se organiza el bombeo de forma que se reducen los consumos de energía.

La sustentabilidad del funcionamiento del sistema también se garantiza con la ejecución de las RDO, pues queda implícito la conservación y protección de la fuente. La sustentabilidad se manifiesta además, dado que la PMI permite evaluar conlos modelos las consecuencias de futuras ampliaciones, cambios tecnológicos, rehabilitaciones $\mathrm{u}$ otras inversiones, antes que se produzcan dichos cambios y dictaminar sobre sus impactos positivos y negativos.

Otros beneficios derivados de la implantación de la PMI se asocian con la adquisición o consolidación de la experiencia práctica de los operadores del sistema en el empleo de los modelos y las herramientas de análisis en el estudio, pronóstico y retroalimentación con la información real de campo obtenida.

La implantación del tercer escalón permite adicionalmente controlar la respuesta en tiempo real del sistema (presiones, abatimientos, conductividad), y adoptar medidas para evitar la ocurrencia de fenómenos perjudiciales, algunos de los cuales pudieran llegar a ser irreversibles o su corrección necesitar importantes desembolsos monetarios.

\section{Tecnología AQÜIMPE}

El software AQÜIMPE desarrollado y aplicado en Cuba, está integrado por un conjunto de programas que realizan la simulación matemática del flujo transitorio y bidimensional en una cuenca subterránea. Sus principales características son las siguientes:

- Utiliza el método de los elementos finitos MEF como técnica numérica empleando el triángulo cuadrático como elemento de discretización, aspecto que lo hace muy eficiente y de fácil adaptación a zonas singulares o de mayor interés.

- Emplea un eficiente método de solución de los sistemas de ecuaciones algebraicas y con un algoritmo que optimiza los requerimientos de memoria, por lo que 
es posible la simulación de grandes sistemas acuíferos utilizando microcomputadoras pequeñas, lo que lo hace ideal para la operación de estos sistemas en las empresas de la producción.

- Simula acuíferos freáticos y confinados, cerrados o abiertos, con descarga al mar simulando el fenómeno de la intrusión salina utilizando una interfaz abrupta.

- Permite simular la interacción entre un cuerpo de agua superficial, dígase un lago o un embalse y el acuífero que lo subyace, siempre que exista un posible intercambio entre ellos (Llanusa y Martínez, 2002).

- La versión actual de AQÜIMPE sobre Windows (WinAQE), permite simular problemas a escala local (pozos, campos de pozos, etc.), aspecto de gran importancia sobre todo en los acuíferos costeros, donde no solo es importante controlar los recursos explotables, sino también dónde y cómo éstos se explotan.

- Las salidas gráficas que ofrece son de gran utilidad para el modelador en cualquier etapa de utilización del modelo, adicionalmente la nueva versión WinAQE, permite la importación y exportación de bases de datos de la versión anterior y hacia otros softwares vinculados a los SIG.

- Se emplea el algoritmo de convergencia global Shuffled Complex Evolution SCE (Gómez, 2009), para la estimación de los parámetros del modelo AQÜIMPE, quedando disponible en la herramienta de software WinAQE

Desde su creación AQÜIMPE ha estado en constante desarrollo. Sin embargo, necesitaba una herramienta que permitiera a los usuarios definir de forma digital la malla de triángulos necesaria para aplicar el MEF, proceso que se realizaba de forma manual, lo cual se tornaba trabajoso, con elevadas posibilidad de cometer errores e imposibilidad de evaluar múltiples variantes, además de la imposibilidad de concebir mallas con zonas más densas de triángulos.

Para resolver este problema se ha creado AQTRIGEO (Cabrera y Escartín, 2008, 2009a y 2009b). Herramienta de un sistema de información geográfica SIG basada en una plantilla del sistema AutoCAD Map, en la cual se incluyen bloques, capas, consultas y macros en Visual Basic para aplicaciones VBA, todos estos son elementos necesarios para la organización y manejo de los datos espaciales de los objetos de la triangulación (nodos, lados y triángulos).
La esencia del proceso se basa en el establecimiento de topologías de polígonos y de puntos a partir de las entidades gráficas que sean creadas. AQTRIGEO tiene una macro que permite definir mallas más densas en la ubicación de pozos de explotación que define el usuario, con las cuales se puede tener en cuenta en la modelación regional los fenómenos locales que afectan a los abatimientos (ver Figura 5).

Todos estos atributos hacen de AQTRIGEO una excelente plataforma para la gestión de los datos espaciales, a un nivel similar a la de sus semejantes en el mundo, pudiéndose resaltar su originalidad científica, sobre todo al emplear el Método de las Componentes Principales para realizar una numeración de los nodos muy eficiente, además de su impacto científico para la tecnología AQÜIMPE ya que por primera vez se define el problema de utilizar los datos de las entidades geométricas que conforman la malla numérica para AQÜIMPE como un problema eminentemente topológico.

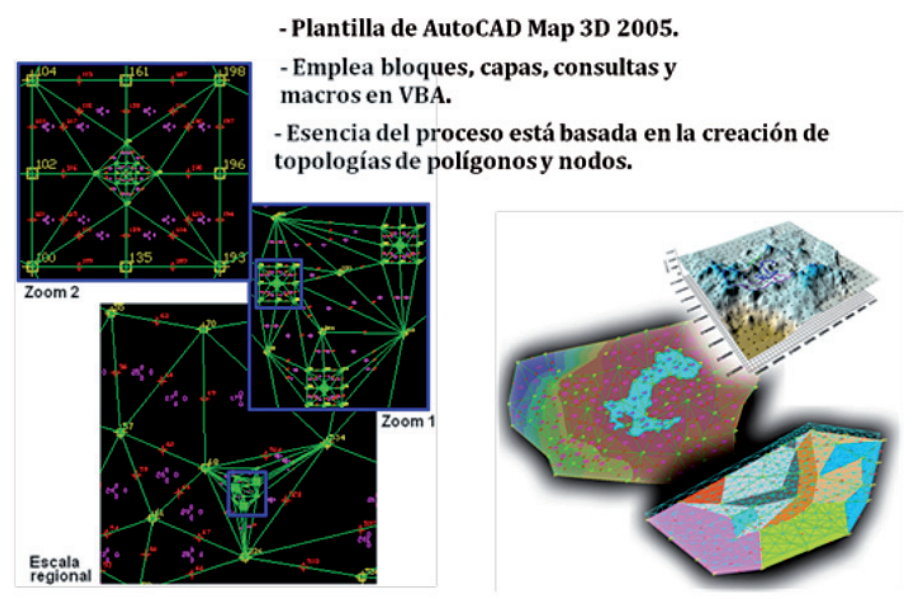

Figura 5: AQTRIGEO, herramienta SIG para el mallado y refinamiento de malla

\section{Ejemplos del empleo de AQüIIMPE}

Desde hace más de tres décadas, en Cuba se ha estado aplicando la tecnología AQUIMPE para la modelación del flujo de las aguas subterráneas con diferentes objetivos, principalmente para evaluar el comportamiento de los acuíferos como respuesta a diferentes obras de ingeniería y en la evaluación de los recursos hidráulicos subterráneos en las zonas estudiadas. No obstante lo anterior, hasta la actualidad no se ha logrado utilizar esta herramienta para 
realizar políticas de asignación de la explotación global ni a corto ni mediano plazo según una primera metodología propuesta por Hernández (1992), donde se presentó un procedimiento para determinar mediante el modelo de simulación los recursos hidráulicos que garantizan el equilibrio entre la recarga anual y la explotación, de cualquier año o conjunto de años. Este procedimiento fue aplicado al modelo "CIRO" en la provincia de Ciego de Ávila y se realiza el pronóstico de la explotación a corto y mediano plazo solo empleando la tecnología de simulación AQÜIMPE.

En la década de 1980 se realizaron diversos proyectos preliminares para la protección contra inundaciones del Aeropuerto Internacional José Martí y la Textilera Ariguanabo, previéndose la construcción de canales de drenaje, rectificación de ríos y la perforación de un número elevado de pozos de recarga subterránea. El objetivo del modelo matemático del acuífero Ariguanabo con un área total de $259.7 \mathrm{~km}^{2}$ fue evaluar la efectividad de tales medidas.

Otro buen ejemplo es el acuífero costero de la Cuenca Sur de La Habana, que actúa como una de las principales fuentes de abasto de la ciudad de la Habana, de las poblaciones enclavadas y de riego de diferentes cultivos, por lo que se construyó a finales de la década del 80 del siglo pasado un dique paralelo a la costa con el objetivo de interrumpir parcialmente el flujo libre hacia el mar de las aguas del humedal e incrementar así el volumen de agua almacenado en el acuífero y la carga hidráulica en la zona de almacenamiento por el efecto de remanso y como consecuencia mejorar la calidad del agua del acuífero. Se planteó la necesidad de confeccionar un modelo para evaluar el efecto del dique abarcando un área de $830 \mathrm{~km}^{2}$. Se ha aplicado en la provincia de Matanzas a la cuenca M-1, en el norte de La Habana en la cuenca Jaruco-Aguacate. También se reporta en Ricardo (2004) su aplicación en la provincia de Las Tunas con el modelo "La Cana".

En otros países cabe destacar la utilización de AQÜIMPE como en México, donde primeramente se realiza la evaluación de la interacción existente entre el lago Tequesquitengo y el acuífero subyacente. Para ello Martínez y Gutiérrez (2004a) calibraron con AQÜIMPE una zona del acuífero que rodea al lago, y después de realizar la simulación, el modelo reprodujo con precisión los niveles observados en el lago demostrándose la importante relación hidráulica que existe entre estos cuerpos de agua (Martínez y Gutiérrez, 2004b). Años después se aplica AQÜIMPE, también en México, pero en este caso en la cuenca del Lago Pátzcuaro donde se demuestra que el lago existe gracias a la presencia de un acuífero que lo subyace y rodea (Cabrera, 2007). Estos trabajos fueron realizados gracias al Instituto Mexicano de Tecnología del Agua IMTA.

\section{Modelación matemática para la evaluación de recursos hidráulicos subterráneos}

Desde hace más de tres décadas en Cuba se ha estado aplicando la tecnología AQÜIMPE para la modelación del flujo de las aguas subterráneas con diferentes objetivos, principalmente para evaluar el comportamiento de los acuíferos como respuesta a diferentes obras de ingeniería y en la evaluación de los recursos hidráulicos subterráneos en las zonas estudiadas. No obstante lo anterior, hasta la actualidad no se ha logrado utilizar esta herramienta para realizar políticas de asignación de la explotación global ni a corto ni mediano plazo según una primera metodología propuesta por Hernández (1992), donde se presentó un procedimiento para determinar mediante el modelo de simulación los recursos hidráulicos que garantizan el equilibrio entre la recarga anual y la explotación, de cualquier año o conjunto de años. Este procedimiento fue aplicado al modelo "CIRO" en la provincia de Ciego de Ávila y se realiza el pronóstico de la explotación a corto y mediano plazo solo empleando la tecnología de simulación AQÜIMPE.

El procedimiento anterior permite evaluar recursos regionales a partir del modelo calibrado y definir estrategias de explotación global, pero sin establecer políticas de explotación óptima, ni considerar restricciones locales, para lo cual se requiere de un modelo de administración que utilice las técnicas de optimización como MADA que se expone a continuación.

\section{Creación del modelo de administración de acuíferos MADA}

El modelo AQÜIMPE resuelve el llamado problema directo de la modelación regional del agua subterránea, es decir, calcula los niveles piezométricos en cada instante 
de tiempo de la simulación en toda la zona de estudio, conocidos previamente, las propiedades hidrogeológicas (por zonas) y los flujos de entrada (fundamentalmente la recarga debido a la lluvia) y de salida (fundamentalmente las extracciones realizadas desde pozos).

Pero AQÜIMPE individualmente no es capaz de resolver los problemas que se plantean a continuación:

- Dada una demanda conocida en el tiempo para todo el acuífero y las capacidades mínima y máxima de extracción de cada pozo del acuífero. ¿Cómo se distribuye espacialmente la extracción de ese volumen de agua para garantizar que al final del período de explotación de los niveles en toda la fuente se encuentren con la menor afectación posible?

- Dadas las capacidades mínima y máxima de extracción de cada pozo del acuífero, así como todas las restricciones de niveles y caudales que garanticen vitalidad y no contaminación de la fuente, ¿Cuál es la máxima cantidad de agua a extraer de la fuente para cada intervalo de tiempo?

Estas y otras interrogantes vitales a resolver para garantizar una administración correcta de los acuíferos, deben ser afrontadas con la ayuda de modelos de simulación como AQÜIMPE, pero deben incluirse otras herramientas que conciban algoritmos de optimización. Este se conoce como uno de los problemas inversos de la modelación regional del agua subterránea.

Para resolver este problema se creó el modelo de administración de acuíferos denominado MADA, el cual se basa en la inclusión de todas las ecuaciones de AQÜIMPE, dentro de un algoritmo de programación lineal como restricciones de igualdad, conocido como sistema acoplado, y este es uno de los aspectos más originales del sistema propuesto. Por lo general esta variante de sistema acoplado no es muy empleada en el mundo, al parecer por su complejidad en la formulación e implementación, sin embargo tiene el valor de tener dentro del algoritmo de optimización al modelo de flujo (en este caso AQÜIMPE), en contraste con el método de la matriz respuesta o Funciones Tecnológicas Algebraicas FTA.

El método de las FTAs fue desarrollado por Dilla (1993), a partir del trabajo desarrollado por Maddock en 1972 y citado por Das y Datta (2001) donde incorpora las respuestas de un modelo de simulación a uno de técnicas de optimización. La propuesta se basa en que es posible construir FTAs que representen linealmente el abatimiento en los pozos de bombeo como función del bombeo o extracción, realizada durante diferentes períodos de tiempo. En Dilla y Mesa (1994) se aplica el método de las FTAs y posteriormente su resultado se introduce en un algoritmo de programación lineal para encontrar políticas óptimas de explotación del acuífero de Ariguanabo en la provincia Habana: maximizar el bombeo en el campo de pozos durante un período húmedo prolongado con restricciones de demanda, límites de caudal de explotación y evitar excesivos descensos, maximizar el bombeo en período seco prolongado y satisfacer la demanda, garantizando la menor depresión del acuífero. Otro aporte en este sentido es publicado por Martínez (2002) donde se utiliza el método de los autovalores con el fin de aplicarlo posteriormente en la generación de FTAs.

En el modelo MADA se desarrollaron un grupo de funciones objetivo de naturaleza lineal, que garantizan diferentes propósitos en la distribución espacial de las extracciones del acuífero, así como restricciones a las variables de decisión que garantizan tener en cuenta aspectos de valor práctico como la imposibilidad de que se pueda extraer de un pozo, más que su caudal máximo asociado a la capacidad instalada. Así se definieron restricciones de demanda, de capacidad instalada y de niveles mínimos.

Otro resultado importante es la introducción en MADA de un procedimiento con el cual se generan un grupo de restricciones que brindan la posibilidad de tener en cuenta todos los efectos locales que modifican los niveles regionales en los pozos de bombeo, y por lo tanto permite que en el proceso de administración se tomen en consideración los niveles reales en los pozos de bombeo (Hernández, 2009; Cabrera y Hernández, 2011). Este procedimiento tiene las ventajas de depender solamente del gasto específico de cada pozo y de emplear una malla pequeña.

\section{Los acuiferos costeros y su gestión}

Algunos de los más diversos y productivos hábitats que engloban extensas áreas de complejos y únicos ecosistemas se encuentran en las áreas costeras; incluyen las planices costeras, zonas litorales, mangles, arrecifes 
coralinos, deltas de ríos, fondos marinos, etc., los cuales son fuentes de producción de alimentos y soporte de una variada actividad económica que incluye la pesca, el turismo, la recreación y la transportación, siendo uno de los principales peligros o amenazas ambientales la fragilidad del equilibrio dinámico que se presentan en los acuíferos costeros por las relaciones entre las aguas dulces, salobres y saladas.

Los países con regiones costeras han reconocido varios elementos que contribuyen a su mayor vulnerabilidad ambiental que incluyen: fragilidad ecológica, rápido crecimiento poblacional humano, limitados recursos de suelos, alta dependencia de los recursos marinos, contaminación por fertilizantes y plaguicidas, exposición a peligros de desastres por eventos extremos y a los cambios climáticos globales, estando muchos de estos factores de vulnerabilidad y sustentabilidad ambiental asociados a las aguas dulces y su principal fuente, los acuíferos costeros. Lo anterior es particularmente cierto en los ecosistemas dependientes de las aguas subterráneas como sucede en las planicies costeras, donde se debe implementar políticas de prevención y remediación, como es la utilización de la recarga artificial en algunos lugares para evitar el avance de la intrusión salina o el drenaje en zonas donde los niveles freáticos altos son amenazas ambientales por la salinización de suelos. Así como facilitar el movimiento de sustancias contaminantes en las aguas subterráneas cuando se reducen los espesores de la zona no satutrada y afectaciones a obras civiles y zonas arqueológicas que que son patrimonio de la humanidad como sucede en la Región la Libertad en la costa del Pacífico de Perú.

Los principales problemas que originan la intrusión salina están asociados a la sobre-explotación del acuífero cuando las demandas exceden las posibilidades de entrega del sistema. En esto incide un inadecuado conocimiento de su dinámica, insuficiente control de la explotación, niveles y calidad del agua, dando lugar a una mala gestión a corto y largo plazo. Los acuíferos costeros constituyen uno de los sistemas más vulnerables a los efectos de la variabilidad climática y al cambio climático. Por ello ha sido objeto de preocupación y análisis por parte de instituciones internacionales y programas de las Naciones Unidas como el Programa Hidrológico Internacional, donde en el VII periodo del 2008-2013 señala la necesidad de atender el impacto del cambio climático en el ciclo hidrológico y su consecuente impacto en los recursos hídricos, por lo que se plantea que el manejo de los sistemas acuíferos deben responder a los cambios globales y a la variabilidad climática. Entre los programas hidrológicos de la UNESCO en acuíferos costeros, cabe destacar GRAPHIC (Groundwater Resources Assessment under the Pressures of Humanity and Climate Change).

Desde la pasada década se viene planteando, la necesidad de enfatizar en el adecuado manejo de los recursos hidráulicos subterráneos costeros en su interacción con las aguas superficiales y los suelos, para garantizar la sostenibilidad de los ecosistemas costeros, sobre todo para enfrentar los problemas de la sequía y otros aspectos de la variabilidad y el cambio climático (Alley et al., 1999). Para lograr el anterior objetivo es necesario, tanto la implementación de nuevas tecnologías, como la utilización adecuada de sistemas de captación y control.

Por lo general la selección del tipo de obras de captación de las aguas subterráneas ha respondido a criterios técnicos, por lo que las más frecuentes utilizadas han sido los pozos verticales. Es importante destacar, que las grandes diferencias entre los niveles estáticos y dinámicos, producidos por los pozos de bombeo verticales (necesariamente de penetraciones parciales por lo que se originan mayores descensos), causa un anormal y rápido crecimiento de la superficie de contacto entre las aguas dulces y las saladas, provocando que altas concentraciones de cloruros permanezcan durante mucho tiempo en las aguas anteriormente dulces (conos de intrusión), antes de regresar a las condiciones naturales originales. La ruptura periódica producto del bombeo de las características del balance de la salinidad de las aguas subterráneas sin un control, lleva a un deterioro acelerado de su calidad hasta convertirse en un problema irreparable a corto plazo.

\section{Aplicación de las nuevas tecnologías en el acuífero costero del sur de La Habana}

El tramo HS-3 de la Cuenca Sur de La Habana se encuentra al sur de la provincia de La Habana y ocupa una superficie aproximada de $830 \mathrm{~km}^{2}$. Es una cuenca cárstica de llanura con cobertura de suelos ferralíticos muy permeables donde hay un intenso desarrollo agrícola.

La hidrogeología de la zona se caracteriza por formaciones con gran heterogeneidad y anisotropía, también se trata de 
una cuenca subterránea abierta al mar por su límite sur donde existe el llamado Dique Sur de La Habana que se muestra en la Figura 6 como una línea de color rojo vino a lo largo de la costa sur.

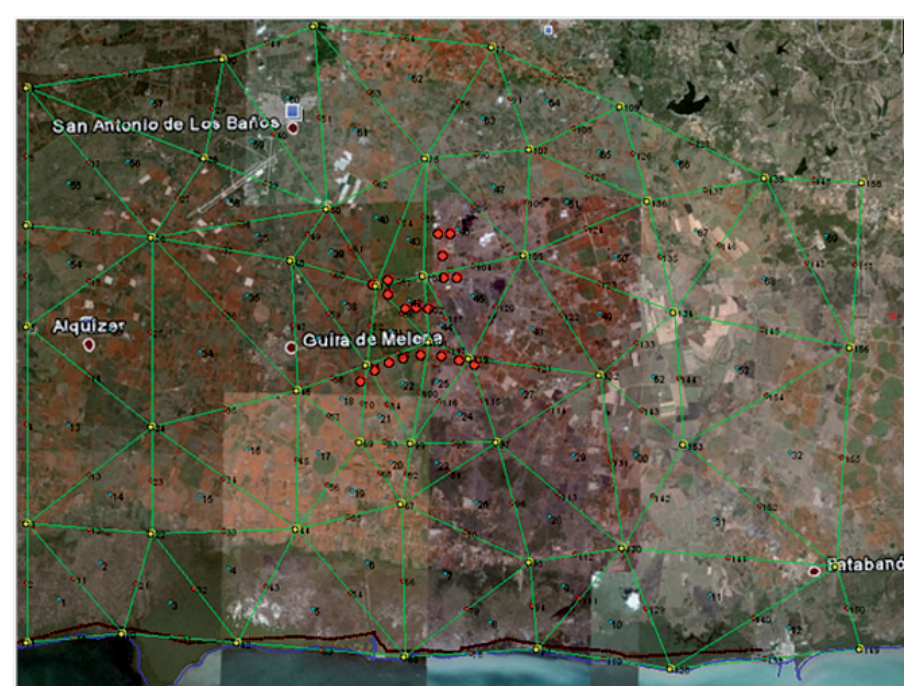

Figura 6: Foto satelital del tramo SH-3 de la Cuenca Sur de la Habana

Esta es una de las zonas más potentes del país desde el punto de vista acuífero y por tal motivo existe una fuerte explotación destinada a la agricultura y al abasto de los principales pueblos de la zona Alquizar-Güira de Melena. Especialmente importante es el caudal que se extrae del llamado campo de pozos Cuenca Sur que se utiliza para el abasto a la ciudad de La Habana y que está constituido por 19 pozos de los que se extrae un caudal de 220 l/s de cada uno, estos pozos se encuentran justo en el centro de la Figura 6, representados por puntos de color rojo.

La implementación de las nuevas tecnologías en este acuífero ha pasado por la utilización de la modelación matemática para evaluar los efectos del Dique Sur, la evaluación de los recursos explotables con las técnicas matemáticas, la introducción de los escalones 1 y 2 de la Plataforma Modular Integrada PMI, la utilización del SCADA para el control y operación de los pozos de bombeo con mando a distancia y la aplicación del modelo de administración MADA para optimizar y definir políticas de explotación atendiendo a los efectos locales en los pozos de bombeo. Se están desarrollando sensores para el control en tiempo real de los niveles en los pozos de bombeo y en pozos de observación, con el objetivo de lograr introducir el escalón 3 de la PMI.
Debido a la intensa explotación de esta zona, y a la inobjetable interacción del acuífero con el mar en la costa sur, existe una cuña de intrusión de agua salada que ha penetrado subterráneamente en tierra alrededor de unos 14 $\mathrm{km}$ bajo esta gran llanura a pesar de la construcción del Dique Sur.

El Dique Sur se construyó precisamente para retener el agua dulce que drenaba superficialmente por una serie de canales que desembocaban en la costa sur, y con esta barrera se crea un nivel de agua dulce que hace retroceder al agua salada. Tiene una cota de corona que varía por zonas de $1.0 \mathrm{~m}$ a $1.5 \mathrm{~m}$ por encima del nivel medio del mar NMM y los aliviaderos están de 0.7 m a 0.9 m del NMM respectivamente.

Con la fuerte explotación de los pozos de Cuenca Sur, para cumplimentar una demanda anual aproximada de 100 $\mathrm{hm}^{3}$, ha habido años de poca lluvia en que algunos pozos se han contaminado por salinización, constituyendo este un aspecto de mucha preocupación para las autoridades encargadas de la administración del preciado recurso.

Es por eso que el objetivo principal de esta primera aplicación del modelo MADA es evaluar la política de explotación de los 19 pozos de Cuenca Sur, tomando como patrón el año 2007, teniendo en cuenta la correcta preservación de los recursos globales del acuífero y una correcta distribución de las extracciones en los pozos.

Primeramente se realizó una amplia búsqueda de información y se prepararon todos los datos necesarios para realizar una simulación de AQÜIMPE teniendo en cuenta el caudal real extraído de los 19 pozos de Cuenca Sur en el año 2007 que ascendió a unos $97.6 \mathrm{hm}^{3}$, el intervalo de tiempo es el mes y se analizaron 12 meses.

Uno de los datos más importantes definidos gracias a una gran cantidad de información brindada por la Empresa Aguas de la Habana fue la relacionada con los pozos de bombeo. En este sentido se pudo definir para cada pozo, el nivel mínimo permisible de carga hidráulica $h_{d}$ por encima del NMM que garantizará según la hipótesis de GhybenHerzberg que la posición del cono de intrusión salina se ubique en el fondo de pozo, ver Figura 7.

En Cabrera (2010) y Cabrera y Dilla (2011) se presentan los resultados de la creación de un sistema para la administración óptima de la explotación del agua 
subterránea en su aplicación a uno de los acuíferos más importantes del país: el tramo HS-3 de la Cuenca Sur de la Habana, donde se evaluó la explotación de los pozos del acueducto Cuenca Sur, incluso teniendo en cuenta recientes pronósticos de los posibles escenarios de eventos extremos y de aumento del nivel medio del mar, derivados del proceso de cambio climático.

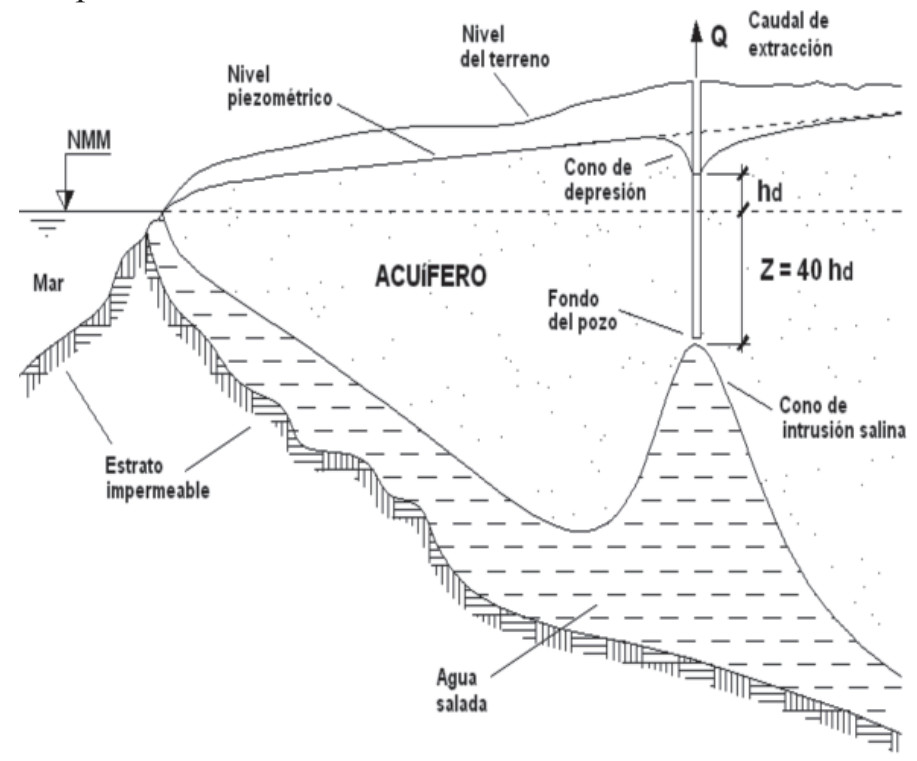

Figura 7: Perfil por el acuífero con cono de intrusión salina provocado por una intensa extracción en los pozos

Los resultados de esta primera simulación indicaron que a nivel global (de todo el acuífero) se podía extraer la cantidad de agua planificada para este año ya que el volumen de agua calculado al final del período de simulación es mayor que el de inicio. Sin embargo, en los pozos 15 y 16 , los niveles reales calculados están muy por debajo de los mínimos permisibles (encontrados como se explicó anteriormente). También es interesante decir que los pozos 12 y 14 no trabajaron en los meses del 3 al 9, posiblemente por roturas y mantenimientos o por extracción de agua con un alto contenido de sal (contaminada). En realidad esto último no se pudo constatar, ya que la información de calidad del agua extraída de cada pozo no fue posible adquirirla a pesar de esfuerzos realizados en este sentido.

Este hecho implica que no solo basta con realizar un balance hidráulico global del acuífero para decidir sobre una correcta política de explotación, sino que es necesario realizar un análisis de la distribución espacial de la explotación.
Con estas ideas se ejecutó el modelo de administración MADA definiendo los niveles mínimos permisibles en cada uno de los pozos como restricciones de nivel, además se empleó la función objetivo que garantiza la máxima extracción del campo de pozos. Los resultados brindan un esquema de bombeo en el cual cada bomba está sin trabajar un mes del año para realizar reparaciones, todos los niveles en los nodos están por encima de los mínimos impuestos e incluso se puede extraer un volumen mayor que el anterior, $106 \mathrm{hm}^{3}$.

Posteriormente se realizó otra aplicación que perseguía el objetivo de evaluar la respuesta del acuífero en el tramo HS-3 de la Cuenca Sur de la Habana ante los posibles escenarios de eventos extremos y de aumento del nivel medio del mar, derivados del proceso de cambio climático. En este sentido los pronósticos de variación interanual de las precipitaciones no son tan precisos, las tendencias observadas durante los últimos años apuntan hacia una intensificación de los períodos de sequía. Por otra parte se pronostican escenarios de cambio climático para Cuba, que indican un aumento del NMM entre $8.87 \mathrm{~cm}$ y $44.41 \mathrm{~cm}$, en dependencia de la sensibilidad climática y el escenario de emisión de gases que se considere, todo esto para un período de 30 años centrado en el 2050.

El acuífero en estudio es de muy rápida respuesta ante los cambios climáticos, como se evidencia en la rapidez con que el mismo se agotó o aumentó su volumen en las corridas de AQÜIMPE, realizadas para los períodos seco y húmedo respectivamente. Esto se debe a que es cárstico y tiene altos valores de transmisividad.

El nivel del remanso de las aguas subterráneas aguas arriba del dique producido por un aumento del NMM, es un factor determinante en la respuesta del acuífero ante esta variación. El nivel del remanso a su vez depende de los niveles en los aliviaderos del dique, por lo tanto es muy importante la revisión y mantenimiento de la estructura del Dique Sur desde el punto de vista de los recursos hidráulicos.

Si se considera un aumento del NMM de $44.41 \mathrm{~cm}$ con respecto al NMM del año 1990 para el período de 30 años centrado en el 2050, y además se supone que las cotas en los aliviaderos del Dique Sur descienden hasta 0.5 m, hipótesis demostrada en Rodríguez (2010), el agua 
del mar penetra en el área de estudio hasta una distancia promedio de $2 \mathrm{~km}$, provocando se inunde una franja de aproximadamente $50 \mathrm{~km}^{2}$.

Para el período de 30 años centrado en el 2050, asumiendo un período climático húmedo y los posibles valores de aumento del NMM de $23.3 \mathrm{~cm}$ y $44.41 \mathrm{~cm}$ correspondientes a las sensibilidades media y alta, es posible extraer el máximo caudal de los pozos del acueducto de Cuenca Sur sin provocar en el acuífero una depresión significativa de sus niveles, ni un avance considerable en la cuña de intrusión salina.

Para el período de 30 años centrado en el 2050, asumiendo un período climático seco, como valor de aumento del NMM $44.41 \mathrm{~cm}$ y que se mantiene el volumen de explotación actual de los pozos para la agricultura y los que abastecen a los distintos pueblos de la provincia Habana, no es posible extraer agua de los pozos del acueducto de Cuenca Sur sin provocar la contaminación de los mismos. Esto implica que para este escenario es necesario replantearse los volúmenes de extracción totales del acuífero, y no solamente los correspondientes a los pozos del acueducto de Cuenca Sur (Rodríguez, 2010).

\section{Conclusiones}

El trabajo muestra que los acuíferos como fuentes de abasto constituyen sistemas muy complejos que requieren del empleo de tecnologías que permitan: i) caracterizarlos mediante modelos conceptuales que integren todas las bases de datos disponibles que permitan conocer su funcionamiento hidrodinámico y respuestas ante diferentes políticas de gestión, ii) la modelación matemática es la única herramienta tecnológica capaz de evaluar el comportamiento de los acuíferos, iii) la confiabilidad de los resultados depende de la calidad de las bases de datos, por lo que se impone mejorar los sistemas de adquisición, almacenamiento y manejo de dichas bases, iv) las políticas de operación deben realizarse bajo diferentes horizontes de planeamiento y no solo para un año, atendiendo a que los sistemas acuíferos por lo general tienen comportamiento hiperanual y, v) las técnicas de optimización deben emplearse para garantizar la mejor explotación del sistema, satisfaciendo las demandas y restricciones necesarias.

La Plataforma Modular Integrada PMI constituye una herramienta capaz de integrar todos los aspectos anteriores, caracterizada por cuatro elementos fundamentales (Gómez, 2009):

1. Integra las tres herramientas que, de manera independiente, se reportan en la literatura para la gestión de los recursos hidráulicos: (I) la Modelación Matemática MM que permite la simulación $\mathrm{y}$ optimización de los procesos a estudiar; (II) el SIG como tecnología informática de la geografía, y que vinculada con la MM, conforman una importante herramienta para el estudio espacial y la gestión de la operación; (III) la implementación de un sistema de adquisición y procesamiento de datos en tiempo real, personalizado para cada aplicación, que tiene en cuenta los procesos de operación y que se encuentra estrechamente vinculado con los resultados que proporcionan los otros dos componentes. Esta integración está basada en datos y un módulo de supervisión general de la operación, que garantiza que el procesamiento de la información relevante aportada por cada componente, sea coherente y efectivo.

2. La información de las variables que describen el proceso, es recibida por diferentes vías y se almacena en una Base de Datos Histórica Actualizada BDHA con diferentes frecuencias en la adquisición del dato y única para todos los componentes de la PMI, lo que posibilita el uso racional de los soportes de almacenamiento, un mejor y más eficiente trabajo de los programas que emplean la BDHA, y una economía importante en la inversión del capital destinado a adquisición y procesamiento de la información que se recolecta. La frecuencia de adquisición de cada variable que integra la BDHA se define en función de la característica temporal de la variable y de la respuesta que el usuario espera del sistema.

3. Está estructurada sobre una estrategia de desarrollo por escalones. Los escalones representan etapas sucesivas que van desde la aplicación de elementos sencillos hasta la introducción de elementos con alto grado de desarrollo tecnológico. De esta manera, cada grupo de resultados (escalón) que se culmina, sirve de sólido apoyo para el siguiente. Por tanto, el proceso inversionista de la solución final estará dividido en etapas y para comenzar el mismo, no es necesario contar con todo el presupuesto necesario para llevar la solución hasta la última. Cada 
escalón es un prerrequisito indispensable del siguiente y un apoyo total, lo cual garantiza que la inversión precedente sea totalmente válida para la siguiente etapa.

4. Con la implementación del primer escalón y hasta la puesta en funcionamiento del último, va en paralelo un proceso de formación de capacidades a todos los niveles que permite a la entidad usuaria adiestrar a su personal para, de esta forma, obtener de la PMI las mayores ventajas en su empleo.

\section{Referencias}

Alley, M., Reilly, E. and Lehn, O. (1999). Sustainability of Ground-Water Resources. U.S. Geological Survey. Circular 1186. Denver, Colorado.

Cabrera, E. (2007). Simulación de acuíferos con el empleo de herramientas de los Sistemas de Información Geográfica. Tesis de Master en Ingeniería Hidráulica, Instituto Superior Politécnico José Antonio Echeverría, Centro de Investigaciones Hidráulicas CIH. La Habana, Cuba.

Cabrera, E. (2010). Sistema para la administración de la explotación del agua subterránea. Tesis de Doctorado, Instituto Superior Politécnico José Antonio Echeverría, Centro de Investigaciones Hidráulicas CIH. La Habana, Cuba.

Cabrera, E., y Escartín, E. (2008). AQTRIGEO, una herramienta SIG para el modelo de flujo en medio poroso (AQÜIMPE). XXIII Congreso Latinoamericano de Hidráulica. Cartagena de Indias, Colombia.

Cabrera, E. y Escartín, E. (2009a). AQTRIGEO Un programa para automatizar el manejo de datos espaciales del modelo bidimensional de flujo de agua subterránea (AQÜIMPE). Manual de usuarios. Instituto Superior Politécnico José Antonio Echeverría, Centro de Investigaciones Hidráulicas CIH, Cuba.

Cabrera, E. y Escartín, E. (2009b). Propuesta para automatizar el manejo de datos espaciales del modelo bidimensional de flujo de agua subterránea (AQÜIMPE). VI Congreso Internacional de Geomática, La Habana, Cuba.

Cabrera, E. y Dilla, F. (2011). Modelo de administración de acuíferos: MADA. Tecnología y Ciencias del Agua 2(4), 1-11.

Cabrera, E. y Hernández, A. (2011). Modelación del agua subterránea a escala regional con refinamiento local de la malla. Planteamiento y validación del algoritmo. Tecnología y Ciencias del Agua 2(1), 65-82.

Das, A. and Datta, B. (2001). Application of optimization techniques in groundwater quantity and quality management. Sadhana 26(4), 293-316.

Dilla, F. y Mesa, H. (1994). Modelos de administración óptima del acuífero Ariguanabo. Boletín Geológico y Minero 105(5), 461-467.

Dilla, F. (1993). Modelación matemática del flujo subterráneo y las técnicas de optimización aplicadas en la investigación y explotación de acuíferos a escala regional. Tesis de Doctorado, Instituto Superior Politécnico José Antonio Echeverría, Centro de Investigaciones Hidráulicas CIH, La Habana, Cuba.

Gómez, M. (2009). Plataforma Modular Integrada para la gestión del agua subterránea como recurso. Tesis de Doctorado, Instituto Superior Politécnico José Antonio Echeverría, Centro de Investigaciones Hidráulicas CIH. La Habana, Cuba.

Hernández, A. (1992). La explotación de acuíferos a escala regional y la modelación matemática como su base tecnológica. Tesis de Doctorado, Instituto Superior Politécnico José Antonio Echeverría, Centro de Investigaciones Hidráulicas CIH. La Habana, Cuba.

Llanusa, H. y Martínez, J.B. (2002). Modelación explícita de embalse y acuífero. Ingeniería Hidráulica en México, 17(4), 89-97

Hernández, A.O. (2009). La modelación matemática y la explotación de los acuíferos costeros. IX Congreso Internacional de Ingeniería Hidráulica, Cuba.

Martínez, J.B. y Gutiérrez, C. (2004a). ¿Por qué ocurren variaciones de nivel en el lago de Tequesquitengol. Calibración de un modelo del sistema hidrológico. Ingeniería Hidráulica en México 19(4), 33-46.

Martínez, J.B. y Gutiérrez, C. (2004b). ¿Por qué ocurren variaciones de nivel en el lago de Tequesquitengo2. Simulación de eventos en el siglo XIX. Ingeniería Hidráulica en México 19(4), 47-56.

Martínez, J.B. (2002). El método de los autovalores en el flujo del agua subterránea. Ingeniería Hidráulica en México 17(3), 37-51.

Ricardo, R. (2004). Modelación del Acuífero La Cana y evaluación de sus Recursos Hidráulicos Subterráneos. Tesis de Master en Ingeniería Hidráulica, Instituto Superior Politécnico José Antonio Echeverría, Centro de Investigaciones Hidráulicas CIH. La Habana, Cuba.

Rodríguez, L. (2010). Posibles impactos del cambio climático en el tramo HS-3 de la Cuenca Sur de La Habana. Tesis de Pregrado en Ingeniería Hidráulica, Instituto Superior Politécnico José Antonio Echeverría, Centro de Investigaciones Hidráulicas CIH. La Habana, Cuba. 